\title{
Pump frequency noise coupling into a microcavity by thermo-optic locking
}

\author{
Jiang Li, ${ }^{1}$ Scott Diddams, ${ }^{2}$ and Kerry J. Vahala ${ }^{1 *}$ \\ ${ }^{1}$ T. J. Watson Laboratory of Applied Physics, California Institute of Technology, \\ Pasadena, CA 91125, USA \\ ${ }^{2}$ Time and Frequency Division, National Institute of Standards and Technology, \\ Boulder, CO 80305, USA \\ *vahala@caltech.edu
}

\begin{abstract}
As thermo-optic locking is widely used to establish a stable frequency detuning between an external laser and a high Q microcavity, it is important to understand how this method affects microcavity temperature and frequency fluctuations. A theoretical analysis of the laser-microcavity frequency fluctuations is presented and used to find the spectral dependence of the suppression of laser-microcavity, relative frequency noise caused by thermo-optic locking. The response function is that of a high-pass filter with a bandwidth and low-frequency suppression that increase with input power. The results are verified using an external-cavity diode laser and a silica disk resonator. The locking of relative frequency fluctuations causes temperature fluctuations within the microcavity that transfer pump frequency noise onto the microcavity modes over the thermal locking bandwidth. This transfer is verified experimentally. These results are important to investigations of noise properties in many nonlinear microcavity experiments in which low-frequency, optical-pump frequency noise must be considered.
\end{abstract}

(C) 2014 Optical Society of America

OCIS codes: (140.4780) Optical resonators; (140.6810) Thermal effects; (140.3945) Microcavities; (143.3325) Laser coupling.

\section{References and links}

1. K. J. Vahala, "Optical microcavities," Nature 424, 839-846 (2003).

2. A. B. Matkso, and V. S. Ilchenko, "Optical resonators with whispering-gallery modes-part I: basics," IEEE J. Quantum Electron. 12, 3-14 (2006).

3. M. L. Gorodetsky, A. A. Savchenkov, and V. S. Ilchenko, "Ultimate Q of optical microsphere resonators," Opt. Lett. 21, 453-455 (1996).

4. D. K. Armani , T. J. Kippenberg, S. M. Spillane, and K. J. Vahala, "Ultra-high-Q toroid microcavity on a chip," Nature 421, 925-928 (2003).

5. H. Lee, T. Chen, J. Li, K. Yang, S. Jeon, O. Painter, and K. J. Vahala, "Chemically etched ultrahigh-Q wedgeresonator on a silicon chip," Nat. Photonics 6, 369-373 (2012)

6. V. S. Ilchenko, and M. L. Gorodetskii, "Thermal nonlinear effects in optical whispering gallery microresonators," Laser Phys. 2, 1004 (1992).

7. A. E. Fomin, M. L. Gorodetsky, I. S. Grudinin, and V. S. Ilchenko, "Nonstationary nonlinear effects in optical microspheres," J. Opt. Soc. Am. B 22, 459-465 (2005).

8. T. Carmon, L. Yang, and K. J. Vahala, "Dynamical thermal behavior and thermal self-stability of microcavities," Opt. Express 12, 4742-4750 (2004).

9. T. J. Kippenberg, H. Rokhsari, T. Carmon, A. Scherer, and K. J. Vahala, "Analysis of Radiation-Pressure Induced Mechanical Oscillation of an Optical Microcavity," Phys. Rev. Lett. 95, 033901, (2005).

10. T. J. Kippenberg, and K. J. Vahala, "Cavity Optomechanics: Back-Action at the Mesoscale," Science 321, 11721176 (2008).

\#209930 - \$15.00 USD Received 10 Apr 2014; revised 30 May 2014; accepted 30 May 2014; published 5 Jun 2014

(C) 2014 OSA $\quad 16$ June 2014 | Vol. 22, No. 12 | DOI:10.1364/OE.22.014559| OPTICS EXPRESS 14559 
11. M. Eichenfield, R. Camacho, J. Chan, K. J. Vahala, and O. Painter, "A picogram-and nanometre-scale photoniccrystal optomechanical cavity," Nature 459, 550-555 (2009).

12. Q. Lin, J. Rosenberg, X. Jiang, K. J. Vahala, and O. Painter, "Mechanical oscillation and cooling actuated by the optical gradient force," Phys. Rev. Lett. 103, 103601 (2009).

13. P. DelHaye, A. Schliesser, O. Arcizet, T. Wilken, R. Holzwarth, and T. Kippenberg, "Optical frequency comb generation from a monolithic microresonator," Nature 450, 1214-1217 (2007).

14. T. J. Kippenberg, R. Holzwarth, and S. A. Diddams, "Microresonator-Based Optical Frequency Combs," Science 322, 555-559 (2011).

15. J. Li, H. Lee, T. Chen, and K. J. Vahala, "Low-Pump-Power, Low-Phase-Noise, and Microwave to MillimeterWave Repetition Rate Operation in Microcombs," Phys. Rev. Lett. 109, 233901 (2012).

16. S. B. Papp and S. A. Diddams, "Spectral and temporal characterization of a fused-quartzmicroresonator optical frequency comb," Phys. Rev. A 84, 053833 (2011).

17. T. Herr, V. Brasch, J. D. Jost, C. Y. Wang, N. M. Kondratiev, M. L. Gorodetsky, and T. J. Kippenberg, “Temporal solitons in optical microresonators," Nat. Photonics 8, 145-152 (2014).

18. J. Li, H. Lee, T. Chen, and K. J. Vahala, "Characterization of a high coherence, Brillouin microcavity laser on silicon," Opt. Express 20, 20170-20180 (2012).

19. J. Li, H. Lee, and K. J. Vahala, “Microwave synthesizer using an on-chip Brillouin oscillator,” Nat. Commun. 4, 2097 (2013).

20. R. Drever, J. L. Hall, F. Kowalski, J. Hough, G. Ford, A. Munley, and H. Ward, "Laser phase and frequency stabilization using an optical resonator," Appl. Phys. B 31, 97-105 (1983).

\section{Introduction}

High-Q microresonators have many applications that result from their combined low optical loss and small mode volume [1-5]. These same properties also mean that thermal effects are readily observable in microcavities. Thermal nonlinear effects in microresonators have been studied previously, including thermal oscillation instability [6,7], thermal linewidth broadening $[6,8]$, and thermal self-stability of cavity resonances [8]. Thermal self-stability of cavity resonances, also called thermo-optic locking, maintains a stable detuning between an external laser and a microcavity resonance [8]. The sign of stable detuning depends upon the sign of $\mathrm{d} L_{O P L} / \mathrm{d} T$ where $L_{O P L}$ is the round-trip optical path length, and in silica a blue-detuned optical signal is stable.

Thermo-optic locking (TOL) makes possible stable optical coupling to a narrow cavity resonance without using any electronic feedback to control the laser frequency [8]. It is widely used in microcavity experiments, including cavity optomechanics [9-12], microresonator-based frequency combs [13-17], microcavity stimulated Brillouin lasers [5,18] and Brillouin microwave synthesis [19]. However, TOL forces the resonant frequency of the microcavity to track any fluctuations in the laser frequency that fall within the thermo-locking bandwidth. It is therefore important to understand this mechanism as it can impact low-frequency fluctuations in these systems.

In this paper, we study the relative frequency noise reduction between an external laser and a microcavity under thermo-optic locking conditions. Using a small signal analysis, we derive the laser-microcavity frequency-detuning fluctuation spectrum and then compare the predictions to measurements of this spectrum using an external cavity diode laser and a $2 \mathrm{~mm}$ silica disk resonator. The transfer of pump noise into other resonator modes is also verified experimentally.

\section{Model of of laser-microcavity frequency noise}

The coupled equations describing the cavity-mode amplitude, $a$, and cavity temperature change, $\Delta T$, can be written as [11],

$$
\begin{aligned}
\dot{a} & =\left[i \Delta-i g_{t h} \Delta T-\frac{\kappa}{2}\right] a+\sqrt{\kappa_{e x}} s \\
\Delta \dot{T} & =-\gamma_{t h} \Delta T+\Gamma|a|^{2}
\end{aligned}
$$

\#209930 - \$15.00 USD Received 10 Apr 2014; revised 30 May 2014; accepted 30 May 2014; published 5 Jun 2014

(C) 2014 OSA 16 June 2014 | Vol. 22, No. 12 | DOI:10.1364/OE.22.014559| OPTICS EXPRESS 14560 
where $a$ is normalized such that $|a|^{2}$ is intracavity field energy, $|s|^{2}$ denotes the input signal power, $\Delta \equiv \omega_{l}-\omega_{o}$ is the laser frequency $\left(\omega_{l}\right)$ detuning relative to the cold cavity resonance $\left(\omega_{o}\right), \kappa\left(\kappa_{e x}\right)$ is the loaded (external) cavity optical energy decay rate. $g_{t h}=-\frac{d n}{d T} \frac{\omega_{o}}{n}$ is the thermo-optic tuning coefficient resulting from thermal changes in the refractive index $(n)$, and $\gamma_{t h}$ is the temperature decay rate. $\Gamma=\Gamma_{a b s} / c_{t h}$ is the heating rate due to optical absorption, where $\Gamma_{a b s}$ is the component of the optical energy dissipation due to material absorption, and $c_{t h}$ is the heat capacity of the volume containing the cavity mode. Equation (2) can be understood as an energy conservation relation: the net heat flow into the cavity (resulting in temperature change) equals the heat flow into the cavity due to optical heating minus the heat flow out of the cavity due to thermal conduction.

Small signal quantities are introduced as follows:

$$
\begin{array}{r}
a=a_{o}+a_{1}(t) \\
\Delta T=\Delta T_{o}+\Delta T_{1}(t) \\
\Delta=\Delta_{0}+\Delta_{1}(t)
\end{array}
$$

where in general $\Delta_{1}(t)$ includes all sources of frequency fluctuation that change the difference of the laser frequency and cavity line center frequency, e.g. laser freqeuency noise, cavity thermorefractive noise and photothermal noise. In this work, the dominant source of these fluctuations is the laser frequency and the intensity noise contributions to the cavity are ignored in the analysis. As an aside, the condition that frequencies of interest (within thermo lock bandwidth) are much slower than the cavity bandwidth also allows introduction of a time-dependent detuning variable in Eq. (5). These give the solutions for steady-state operation:

$$
\begin{array}{r}
a_{o}=\sqrt{\kappa_{e x}} s /\left(-i\left[\Delta_{o}-g_{t h} \Delta T_{o}\right]+\frac{\kappa}{2}\right) \\
\Delta T_{o}=\frac{\Gamma\left|a_{o}\right|^{2}}{\gamma_{t h}}=\frac{\Gamma}{\gamma_{t h}} \frac{\kappa_{e x}|s|^{2}}{\left[\Delta_{o}-g_{t h} \Delta T_{o}\right]^{2}+\kappa^{2} / 4}
\end{array}
$$

and, upon linearization, the following time-dependent coupled system:

$$
\begin{aligned}
a_{1} & =\left[i \Delta_{o}-i g_{t h} \Delta T_{o}-\frac{\kappa}{2}\right] a_{1}+i\left[\Delta_{1}-g_{t h} \Delta T_{1}\right] a_{o} \\
\Delta \dot{T}_{1} & =-\gamma_{t h} \Delta T_{1}+\Gamma\left(a_{o} a_{1}^{*}+a_{o}^{*} a_{1}\right)
\end{aligned}
$$

Solving Eq. (7) gives the typical thermal bistability tuning curve of a microcavity [8]. Because the cavity field damping rate, $\kappa / 2$, is typically much faster than the thermal damping rate of the cavity, $\gamma_{t h}$, the steady-state form of Eq. (8) can be used throughout the analysis (i.e., field adiabatically follows the mode temperature and laser frequency fluctuations at rates less than the cavity bandwidth):

$$
a_{1}=\frac{i\left[\Delta_{1}-g_{t h} \Delta T_{1}\right] a_{o}}{-i\left[\Delta_{o}-g_{t h} \Delta T_{o}\right]+\frac{\kappa}{2}}
$$

Upon substitution of Eq. (10) into Eq. (9), the following equation results for the temperature fluctuations of the optical mode driven by fluctuations of the laser detuning, $\Delta_{1}$ :

$$
\Delta \dot{T}_{1}=-\left[\gamma_{t h}-\frac{2 \Gamma g_{t h}\left|a_{o}\right|^{2}\left(\Delta_{o}-g_{t h} \Delta T_{o}\right)}{\left(\Delta_{o}-g_{t h} \Delta T_{o}\right)^{2}+\frac{\kappa^{2}}{4}}\right] \Delta T_{1}-\frac{2 \Gamma\left|a_{o}\right|^{2}\left(\Delta_{o}-g_{t h} \Delta T_{o}\right)}{\left(\Delta_{o}-g_{t h} \Delta T_{o}\right)^{2}+\frac{\kappa^{2}}{4}} \Delta_{1}
$$

Solving this equation in the frequency domain gives,

$$
\Delta \tilde{T}_{1}(\Omega)=\frac{-\frac{2 \Gamma\left|a_{o}\right|^{2}\left(\Delta_{o}-g_{t h} \Delta T_{o}\right)}{\left(\Delta_{o}-g_{t h} \Delta T_{o}\right)^{2}+\frac{\kappa^{2}}{4}}}{\gamma_{t h}-\frac{2 \Gamma g_{t h}\left|a_{o}\right|^{2}\left(\Delta_{o}-g_{t h} \Delta T_{o}\right)}{\left(\Delta_{o}-g_{t h} \Delta T_{o}\right)^{2}+\frac{\kappa^{2}}{4}}+i \Omega} \tilde{\Delta}_{1}(\Omega)
$$

\#209930 - \$15.00 USD Received 10 Apr 2014; revised 30 May 2014; accepted 30 May 2014; published 5 Jun 2014 (C) 2014 OSA 16 June 2014 | Vol. 22, No. 12 | DOI:10.1364/OE.22.014559 | OPTICS EXPRESS 14561 


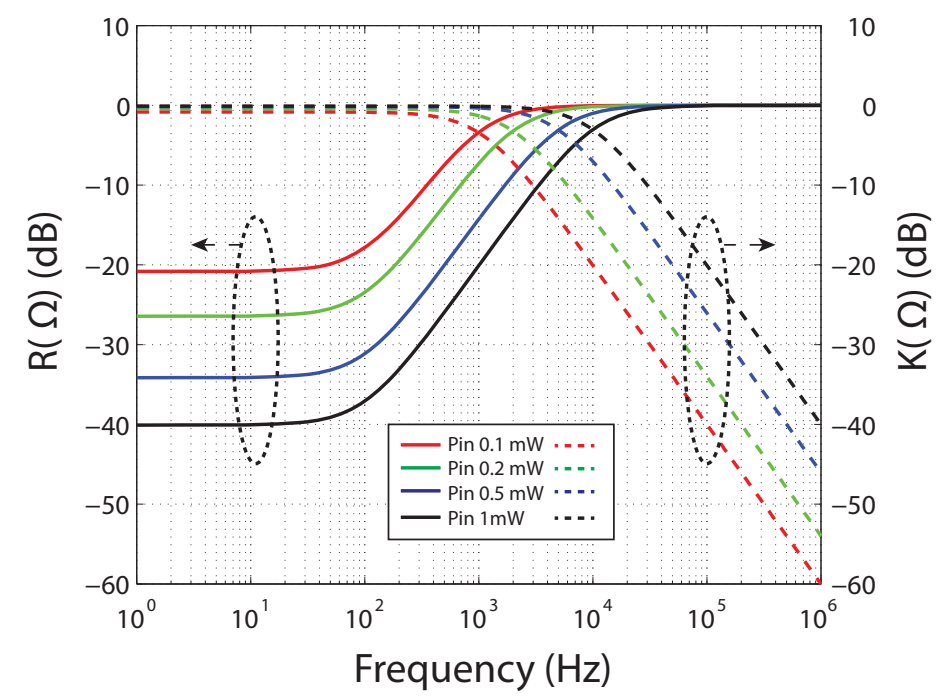

Fig. 1. Plot of thermo-optic lock, laser-microcavity, relative-frequency-noise transfer function $(R(\Omega))$ and the cavity-frequency-noise transfer function $(K(\Omega))$ for several input laser power levels at a warm-cavity detuning of $\Delta_{o}-g_{t h} \Delta T_{o}=\kappa / 2$. Parameters used in these plots are $\gamma_{t h} / 2 \pi=100 \mathrm{~Hz}$, and $\alpha / 2 \pi=10 \mathrm{kHz} / \mathrm{mW}$.

On the other hand, the laser-microcavity relative frequency fluctuation in the frequency domain is

$$
\tilde{\Delta}_{1}^{l c}(\Omega)=-g_{t h} \Delta \tilde{T}_{1}(\Omega)+\tilde{\Delta}_{1}(\Omega)
$$

Substituting Eq. (12) into Eq. (13), the spectral density of the laser-microcavity relative frequency fluctuation, $S_{\Delta \omega}^{l c}(\Omega) \equiv\left|\tilde{\Delta}_{1}^{l c}(\Omega)\right|^{2}$, is given by,

$$
S_{\Delta \omega}^{l c}(\Omega)=\frac{\gamma_{t h}^{2}+\Omega^{2}}{\left[\gamma_{t h}+\alpha P_{i n}\right]^{2}+\Omega^{2}} S_{\Delta \omega}(\Omega)
$$

where

$$
\alpha=\frac{d n}{d T} \frac{\omega_{o}}{n} \Gamma \frac{2 \kappa_{e x}\left(\Delta_{o}-g_{t h} \Delta T_{o}\right)}{\left[\left(\Delta_{o}-g_{t h} \Delta T_{o}\right)^{2}+\frac{\kappa^{2}}{4}\right]^{2}}
$$

and $S_{\Delta \omega}(\Omega) \equiv\left|\tilde{\Delta_{1}}(\Omega)\right|^{2}$ is the spectral density of the free-running laser frequency fluctuations. Note that $\Delta_{o}-g_{t h} \Delta T_{o}$ is the steady-state detuning for the warm cavity. We can define the TOL laser-microcavity relative frequency noise transfer function as:

$$
R(\Omega)=\frac{\gamma_{t h}^{2}+\Omega^{2}}{\left[\gamma_{t h}+\alpha P_{i n}\right]^{2}+\Omega^{2}}
$$

This expression has the form of a high-pass filter response governed by a zero at $\gamma_{t h}$ and a power-dependent pole at $\gamma_{t h}+\alpha P_{i n}$. The transfer function is plotted in Fig. 1 for a range of input laser power levels at a constant detuning. On account of the power-dependent pole, there can be a substantial suppression of the noise. It is worth commenting on the impact of cavity $\mathrm{Q}$ (or photon damping rate $\kappa$ ) on the TOL bandwidth and frequency-noise suppression. As $\kappa_{e x}$ and $\Delta_{o}-g_{t h} \Delta T_{o}$ are both typically on the order of $\kappa$, it follows that $\alpha \sim 1 / \kappa^{2}$. Therefore, a high optical Q greatly enhances the TOL bandwidth and frequency noise suppression factor.

\#209930 - \$15.00 USD Received 10 Apr 2014; revised 30 May 2014; accepted 30 May 2014; published 5 Jun 2014 (C) 2014 OSA 16 June 2014 | Vol. 22, No. 12 | DOI:10.1364/OE.22.014559 | OPTICS EXPRESS 14562 
Physically, $R(\Omega)$ at $\Omega=0$ captures the so-called thermal-locking behavior of a high-Q microcavity on account of the temperature dependence of refractive index. In addition, however, the frequency response contained in $R(\Omega)$ shows how this locking behavior is bandwidth limited to a range determined by the thermal damping rate.

While the laser-microcavity, relative frequency fluctuations are suppressed due to thermooptic lock, the absolute frequency of cavity and the mode temperature of the cavity experience fluctuations from the laser as a result of the locking. The microcavity temperature fluctuation spectrum can be derived from Eq. (12):

$$
S_{\Delta T}(\Omega)=\frac{\left[\alpha P_{i n}\right]^{2} / g_{t h}^{2}}{\left[\gamma_{t h}+\alpha P_{i n}\right]^{2}+\Omega^{2}} S_{\Delta \omega}(\Omega)
$$

Accordingly, the cavity resonance frequency fluctuation spectrum is given by:

$$
S_{\Delta \omega}^{c a v}(\Omega)=g_{t h}^{2} S_{\Delta T}(\Omega)=\frac{\left[\alpha P_{i n}\right]^{2}}{\left[\gamma_{t h}+\alpha P_{i n}\right]^{2}+\Omega^{2}} S_{\Delta \omega}(\Omega)
$$

We define the TOL cavity frequency noise transfer function as:

$$
K(\Omega)=\frac{\left[\alpha P_{i n}\right]^{2}}{\left[\gamma_{t h}+\alpha P_{i n}\right]^{2}+\Omega^{2}}
$$

$K(\Omega)$ is a low pass filter function (a power dependent pole at $\gamma_{t h}+\alpha P_{i n}$ ) that gives the transfer of laser frequency noise into cavity frequency noise. The transfer function is also plotted in Fig. 1 (right vertical axis) for a series of input laser power levels at constant detuning. It can be seen that an increase of the input laser power not only gives a higher suppression of lasermicrocavity relative frequency fluctuations, it also causes the microcavity resonance to track the external input laser more tightly with a larger bandwidth.

\section{Measurement of laser-microcavity relative-frequency-noise suppression}

The laser-microcavity relative-frequency-noise is contained in the power fluctuations transmitted past the cavity. Specifically, the cavity resonance lineshape acts as a frequency discriminator to convert the laser-microcavity, relative-frequency noise to power fluctuations in the transmitted signal. Therefore the power spectral density of the cavity transmission, $S_{P}(\Omega)$, is related to the power spectral density of the laser-microcavity frequency noise $S_{\Delta \omega}^{l c}(\Omega)$ as follows:

$$
S_{P}(\Omega)=P_{i n}^{2} H(\Omega) S_{\Delta \omega}^{l c}(\Omega)
$$

where $P_{i n}$ is the input power and $H(\Omega)$ is the cavity frequency discrimination function. Here we neglect the laser intensity noise contributions to the output power fluctuations. $H(\Omega)$ has the following form [12],

$$
H(\Omega)=\frac{\kappa_{e x}^{2}}{\left[\Delta^{2}+\kappa^{2} / 4\right]^{2}} \frac{4 \Delta^{2}\left(\kappa_{i}^{2}+\Omega^{2}\right)}{\left[(\Delta+\Omega)^{2}+\kappa^{2} / 4\right]\left[(\Delta-\Omega)^{2}+\kappa^{2} / 4\right]}
$$

For Fourier frequencies much less than the cavity linewidth, $H(\Omega) \approx H_{o}=\frac{4 \kappa_{x}^{2} \kappa_{i}^{2} \Delta^{2}}{\left[\Delta^{2}+\kappa^{2} / 4\right]^{4}}$.

In the measurement, a resonance in a $2 \mathrm{~mm}$ disk resonator [5] with loaded linewidth of 4.1 $\mathrm{MHz}(\mathrm{Q}=47$ million) at critical coupling is thermo-optically locked using an external cavity diode laser (ECDL). A series of input power levels (from $25 \mu \mathrm{W}$ to $2 \mathrm{~mW}$ ) are applied with the laser detuned to the half-linewidth point (i.e., $\Delta=\kappa / 2$ ). The measured lasermicrocavity, relative-frequency-noise spectra are given in Fig. 2(a). Note that $S_{\Delta v}^{l c} \equiv S_{\Delta \omega}^{l c} / 4 \pi^{2}$

\#209930 - \$15.00 USD Received 10 Apr 2014; revised 30 May 2014; accepted 30 May 2014; published 5 Jun 2014 (C) 2014 OSA 16 June 2014 | Vol. 22, No. 12 | DOI:10.1364/OE.22.014559 | OPTICS EXPRESS 14563 

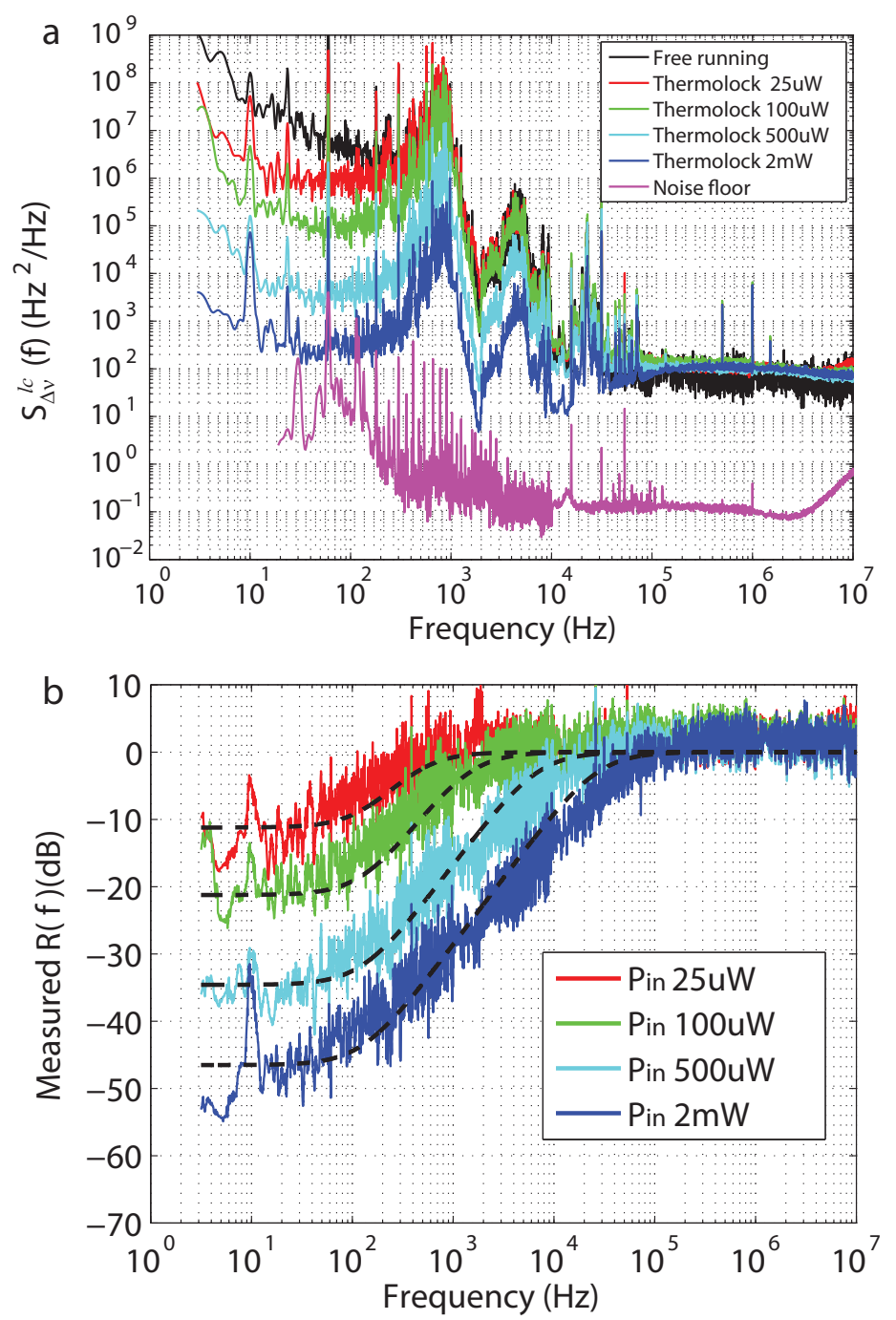

Fig. 2. (a) The laser-microcavity relative-frequency-noise spectra are measured for a $2 \mathrm{~mm}$ disk resonator with loaded linewidth $4.10 \mathrm{MHz}$ under a series of input power levels: $25 \mu \mathrm{W}$ (red curve), $100 \mu \mathrm{W}$ (green curve), $500 \mu \mathrm{W}$ (cyan curve) and $2 \mathrm{~mW}$ (blue curve). For comparison, the free-running laser frequency noise is also shown (black curve), measured using a Mach-Zehnder interferometer [5]. The magenta curve is the background noise when the laser is off-resonance and contains the laser relative intensity noise (RIN) and shot noise normalized by the collected power and transfer function $H$. Background noise (magenta curve) rises above $4 \mathrm{MHz}$ is a result of the shot noise divided by the cavity transfer function. Note that the spurious noise at low frequencies (e.g. at $10 \mathrm{~Hz}$ ) is believed to result from corresponding peaks in the frequency noise (e.g. mechanical noise) of the pump laser. (b) TOL laser-microcavity relative-frequency-noise transfer function, $R$, is plotted at a series of input power levels given in (a). The black-dashed curves are calculations based on Eq. (16) using the following fitting parameters $\gamma_{t h} / 2 \pi=128 \mathrm{~Hz}$ and $\alpha / 2 \pi=13.5 \mathrm{kHz} / \mathrm{mW}$. TOL-induced laser-microcavity relative-frequency-noise suppression increases with the input power as does the TOL bandwidth.

\#209930 - \$15.00 USD Received 10 Apr 2014; revised 30 May 2014; accepted 30 May 2014; published 5 Jun 2014 (C) 2014 OSA 16 June 2014 | Vol. 22, No. 12 | DOI:10.1364/OE.22.014559 | OPTICS EXPRESS 14564 


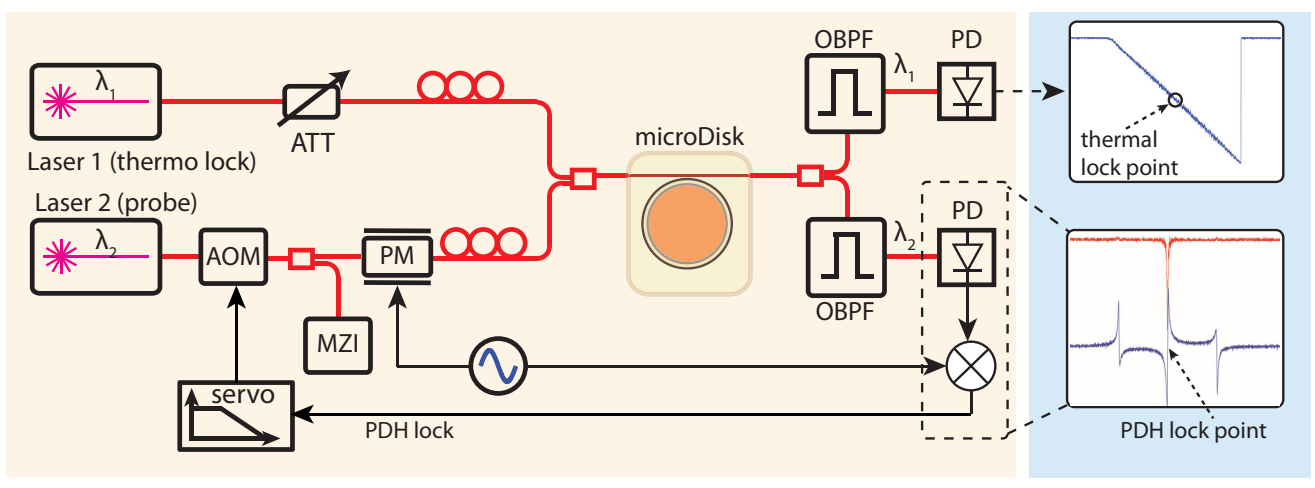

Fig. 3. Experimental layout is given for the pump-probe experiment to measure the cavity frequency noise induced by thermo-optic locking. Laser 1 is an external cavity diode laser, which is thermo-optically locked to a microcavity mode at the half-detuning point. Laser 2 is a narrow linewidth, tunable fiber laser, which is locked to a different cavity mode using Pound-Drever-Hall (PDH) technique. The fiber laser is also coupled to a Mach-Zehnder interferometer (MZI) for laser frequency noise measurement. AOM: acousto-optic modulator; ATT: tunable optical attenuator; PM: phase modulator; OBPF: optical band pass filter; PD: photodetector.

as $\Delta v=\Delta \omega / 2 \pi$. The magenta curve in Fig. 2(a) is the background noise when the laser is off-resonance and contains the laser relative intensity noise (RIN) and shot noise normalized by the collected power and transfer function $H$. The rise of the background noise above $4 \mathrm{MHz}$ is due to the division of shot noise to the cavity transfer function $H$. (Here it can be seen that the dominant noise source of the transmitted (or intracavity) power fluctuations for a high Q cavity is from the laser frequency noise. ) Also plotted in black is the free-running laser frequency noise measured independently using a Mach-Zehnder interferometer (MZI) as a frequency discriminator [5]. (Note that the transfer function of the MZI is given for the measurement of laser frequency noise in [5].) Below $1 \mathrm{kHz}$ the noise is suppressed by an amount that increases with input power. The same spectra normalized by the free-running laser frequency noise give the TOL laser-microcavity relative-frequency-noise transfer function $R$ (see Eq. (16)) and are plotted in Fig. 2(b). For comparison a series of theoretical curves (dashed black curves) based on the model in the previous section are also plotted using the following fitting parameters $\gamma_{t h} / 2 \pi=$ $128 \mathrm{~Hz}$, and $\alpha / 2 \pi=13.5 \mathrm{kHz} / \mathrm{mW}$. The data in Fig. 2(b) make clear the increasing suppression of noise and also increasing bandwidth with higher input power.

\section{Cavity frequency noise induced by a thermo-locked laser}

As discussed above, thermo-optic locking causes the cavity resonance to track the external laser frequency fluctuations within the thermo-optic bandwidth. This tracking will also transfer to other modes of the microcavity that have similar spatial profiles (i.e., similar whisperinggallery transverse mode families). In order to measure the cavity frequency noise induced by a laser that is thermo-optically locked to the microcavity, a pump-probe method is used as shown in Fig. 3. The pump laser is an external cavity diode laser (ECDL) shown as laser 1, and the probe laser is a narrow linewidth tunable fiber laser (FL) shown as laser 2. In the measurement, the pump laser is thermo-optically locked at the half detuning point to the same cavity mode in section 3 and the probe laser is locked to the line center of a different cavity mode using the Pound-Drever-Hall technique [20]. This other mode is located two FSR (66 GHz) away from the thermo-locked mode and belongs to the same spatial mode family. A portion of the

\#209930 - \$15.00 USD Received 10 Apr 2014; revised 30 May 2014; accepted 30 May 2014; published 5 Jun 2014 (C) 2014 OSA 16 June 2014 | Vol. 22, No. 12 | DOI:10.1364/OE.22.014559| OPTICS EXPRESS 14565 
a

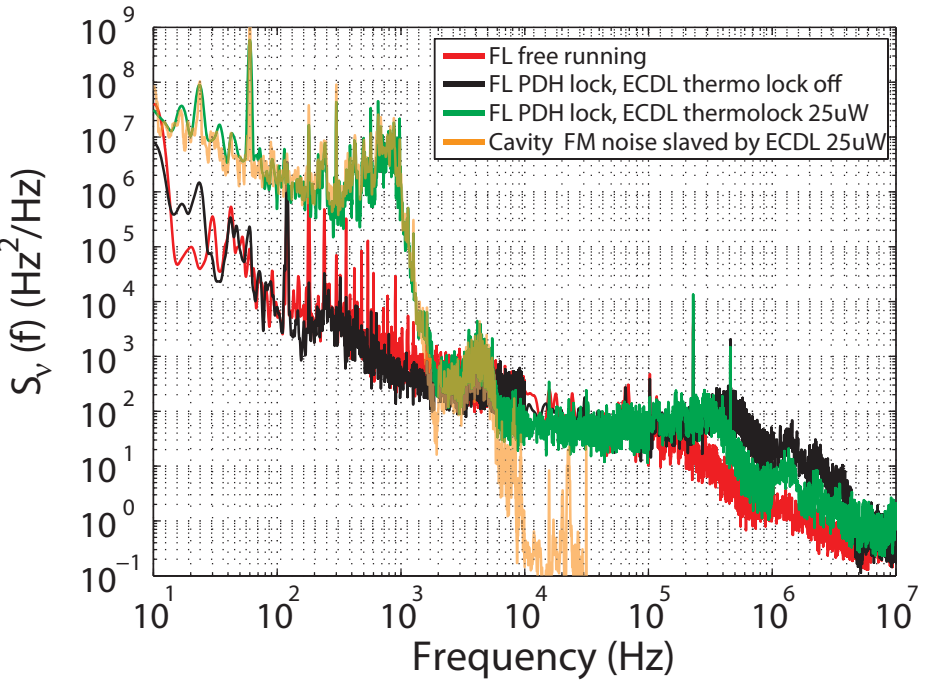

b

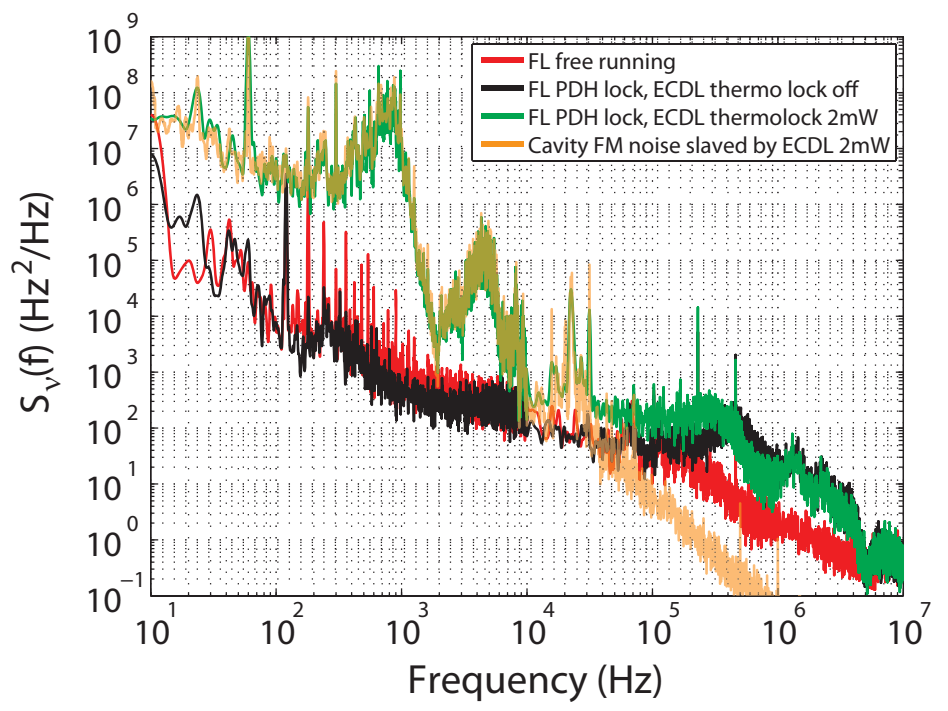

Fig. 4. (a) Shown in the figure are the measured PDH-locked fiber laser (FL) frequency noise spectrum (green curve) and the predicted frequency noise spectrum of the thermallyslaved cavity pumped by an ECDL with $25 \mu \mathrm{W}$ power (brown curve). Also plotted for comparison are the PDH-locked fiber laser frequency noise without ECDL thermo-optic locking (black) and the free-running fiber laser frequency noise (red). (b) The measured PDH-locked fiber laser frequency noise spectrum (green curve) and the predicted microcavity frequency noise spectrum induced by the thermo-optically locked ECDL with $2 \mathrm{~mW}$ power (brown curve) are shown.

\#209930 - \$15.00 USD Received 10 Apr 2014; revised 30 May 2014; accepted 30 May 2014; published 5 Jun 2014 (C) 2014 OSA 16 June 2014 | Vol. 22, No. 12 | DOI:10.1364/OE.22.014559| OPTICS EXPRESS 14566 
PDH-locked probe laser is also sent to a MZI for frequency noise measurement. The probe laser power is kept constant and low $(\sim 10 \mu \mathrm{W})$ and the pump laser power is varied from 25 $\mu \mathrm{W}$ to $2 \mathrm{~mW}$. As a sidenote, because the probe laser is locked to the cavity line center (zero detuning), there is no thermo-optic locking effect as shown by Eq. (15). Thus the frequency noise of the PDH-locked probe laser represents the cavity frequency noise within the PDH locking bandwidth.

The measured laser frequency noise spectra of the PDH-locked fiber laser are plotted in Fig. 4 , for ECDL power levels of $25 \mu \mathrm{W}$ (green curve in Fig. 4(a)) and $2 \mathrm{~mW}$ (green curve in Fig. 4(b)). Also plotted for comparison are the free-running fiber-laser frequency noise (red curve) and the PDH-locked fiber laser frequency noise without the ECDL in thermo-optic lock (black curve). The servo bump for PDH lock is located around $300 \mathrm{kHz}$. An increase of the PDH-locked fiber laser frequency noise can be seen with the increase of the power levels of the thermo-optically locked ECDL from these two figures. Using the fitting parameters, $\gamma_{\text {th }}$ and $\alpha$, and the measured ECDL frequency noise obtained in section 3, the expected cavity frequency noise induced by the thermo-optic locking process is also shown in the figures (brown curve) by using Eq. (18). The measured PDH-locked fiber frequency noise agree very well with the predicted frequency noise of a thermally-slaved cavity based on the transfer function $K(\Omega)$ at low offsets, and they are much higher than the free-running FL frequency noise or the frequency noise of a PDH-locked fiber laser on a "cold" cavity. On the other hand, at higher frequencies the PDH-locked fiber laser FM noise is mainly limited by fiber laser's own frequency noise in the measurement.

\section{Conclusion}

As thermo-optic locking is widely used in microcavity experiments, it is important to understand how this method affects the laser-microcavity relative-frequency-fluctuations and absolute frequency fluctuations of the microcavity. We have presented a theoretical analysis of the laser-microcavity frequency fluctuations under thermo-optic locking conditions. The transfer function governing coupling of laser frequency fluctuations into relative frequency fluctuations of the laser-microcavity system is a high-pass-filter response whose bandwidth varies linearly with power and low-frequency fluctuation suppression varies quadratically with power. We also measured the relevant frequency fluctuation spectra using an external-cavity diode laser and a 2 $\mathrm{mm}$ silica disk resonator. The measured spectra are in good agreement with the model. Within the thermal locking bandwidth, there is efficient coupling of laser frequency fluctuations into the microcavity temperature fluctuations and also the microcavity resonant frequencies. These studies will be useful in studying the impact of pump frequency noise on measurements in a range of subjects including cavity optomechanics, microresonator-based frequency combs and microcavity Brillouin lasers.

\section{Acknowledgments}

This work is supported by the DARPA ORCHID and QUASAR programs. The authors are also grateful for the support from the Institute for Quantum Information and Matter, an NSF Physics Frontiers Center with support of the Gordon and Betty Moore Foundation, and the Kavli NanoScience Institute. J.L. acknowledges the support from the Kavli Nanoscience Institute for a postdoctoral fellowship.

\#209930 - \$15.00 USD Received 10 Apr 2014; revised 30 May 2014; accepted 30 May 2014; published 5 Jun 2014 (C) 2014 OSA 16 June 2014 | Vol. 22, No. 12 | DOI:10.1364/OE.22.014559| OPTICS EXPRESS 14567 